\title{
Well-posedness for a class of generalized variational-hemivariational inequalities involving set-valued operators
}

\section{Caijing Jiang ${ }^{1 *}$}

\section{"Correspondence:}

jiangcaijing@outlook.com

${ }^{1}$ College of Sciences, Guangxi

University for Nationalities, Nanning,

P.R. China

\section{Springer}

\begin{abstract}
The aim of present work is to study some kinds of well-posedness for a class of generalized variational-hemivariational inequality problems involving set-valued operators. Some systematic approaches are presented to establish some equivalence theorems between several classes of well-posedness for the inequality problems and some corresponding metric characterizations, which generalize many known results. Finally, the well-posedness for a class of generalized mixed equilibrium problems is also considered.
\end{abstract}

Keywords: Generalized variational-hemivariational inequality; Set-valued operator; $\alpha$-well-posedness; Monotonicity

\section{Introduction}

Nowadays, well-posedness has been drawing great attention in the field of optimization problems and related problems such as variational inequalities, hemivariational inequalities, fixed point problems, equilibrium problems, and inclusion problems (see $[1,5,9,11$, $17,19,21,23,33])$. The classical concept of well-posedness for a global minimization problem was first introduced by Tikhonov [35], which required the existence and uniqueness of a solution to the global minimization problem and the convergence of every minimizing sequence toward the unique solution. Thereafter, the concept of well-posedness has been generalized to variational inequalities. The initial notion of well-posedness for variational inequality is due to Lucchetti and Patrone [28]. Fang [13, 14] generalized two kinds of well-posedness for a mixed variational inequality problem in a Banach space. For further results on the well-posedness of variational inequalities, we refer to $[2,4,12-14,16,22$, $27,28]$ and the references therein.

As an important and useful generalization of variational inequality, hemivariational inequality, which was first studied by Panagiotopoulos [32], has a great development in recent years by several works $[6,29,31]$. Many authors are interested in generalizing the concept of well-posedness to hemivariational inequalities. In 1995, Goeleven and Mentagui [15] generalized the concept of the well-posedness to a hemivariational inequality and presented some basic results concerning the well-posed hemivariational inequality. Recently, using the concept of approximating sequence, Xiao et al. [37, 38] introduced a concept of well-posedness for a hemivariational inequality and a variational-hemivariational

(c) The Author(s) 2018. This article is distributed under the terms of the Creative Commons Attribution 4.0 International License (http://creativecommons.org/licenses/by/4.0/), which permits unrestricted use, distribution, and reproduction in any medium, provided you give appropriate credit to the original author(s) and the source, provide a link to the Creative Commons license, and indicate if changes were made. 
inequality. Ceng, Lur, and Wen [3] considered an extension of well-posedness for a minimization problem to a class of generalized variational-hemivariational inequalities with perturbations in reflexive Banach spaces. For more recent works on the well-posedness for variational-hemivariational inequalities, we refer to $[3,15,18,19,26,37,38]$ and the references therein.

In the last years, many authors studied the existence results for some types of hemivariational inequalities involving set-valued operators [34, 36, 39]. In 2011, Zhang and He [39] studied a kind of hemivariational inequalities of Hartman-Stampacchia type by introducing the concept of stable quasimonotonicity. They supposed that the constraint set is a bounded (or unbounded), closed, and convex subset in a reflexive Banach space. The authors gave sufficient conditions for the existence and boundedness of solutions. In 2013, Tang and Huang [34] generalized the result of [39] by introducing the concept of stable $\phi$-quasimonotonicity and obtained some existence theorems when the constrained set is nonempty, bounded (or unbounded), closed, and convex in a reflexive Banach space. Hereafter, Wangkeeree and Preechasilp [36] generalized the results of [34] and [39] by introducing the concept of stable $f$-quasimonotonicity. Very recently, Liu and Zeng obtained some existence results for a class of hemivariational inequalities involving the stable $(g, f, \alpha)$-quasimonotonicity [25], a result on the well-posedness for mixed quasivariational hemivariational inequalities [26], and some existence results for a class of quasimixed equilibrium problems involving the $(f, g, h)$-quasimonotonicity [24].

Let $K$ be a nonempty, closed, and convex subset of a real Banach space $X$ with its dual $X^{*}$, and let $F: K \rightarrow P\left(X^{*}\right)$ be a set-valued operator, where $P\left(X^{*}\right)$ is the set of all nonempty subsets of $X^{*}$. Let $T: K \rightarrow X^{*}$ be a perturbation, and let $f \in X^{*}$ be a given element. Let $g: K \times K \rightarrow \bar{R}:=R \cup\{ \pm \infty\}$ be a function such that $\mathcal{D}(g)=\{u \in K: g(u, v) \neq-\infty, \forall v \in K\} \neq$ $\emptyset$. Let $J: X \rightarrow R$ be a locally Lipschitz function, and let $J^{\circ}(u, v)$ denote the generalized directional derivative in the sense of Clarke of a locally Lipschitz functional $J: X \rightarrow R$ at $u$ in the direction $v$. In this paper, we discuss the following generalized variationalhemivariational inequality (GVHVI):

Find $u \in K$ such that, for some $u^{*} \in F(u)$,

$$
\left\langle u^{*}+T u-f, v-u\right\rangle+g(u, v)+J^{\circ}(u ; v-u) \geq 0, \quad \forall v \in K .
$$

Now, let us consider some particular cases of GVHVI.

(a) If $T \equiv 0, f \equiv 0$, and $g \equiv 0$, then GVHVI is reduced to the following form:

Find $u \in K$ and $u^{*} \in F(u)$ such that

$$
\left\langle u^{*}, v-u\right\rangle+J^{\circ}(u ; v-u) \geq 0, \quad \forall v \in K .
$$

The existence of solutions to this inequality was recently studied by Zhang and $\mathrm{He}$ [39].

(b) If $T \equiv 0$ and $f \equiv 0$, and $g(u, v)=\phi(v)-\phi(u)$ for all $u, v \in K$, then GVHVI is reduced to the following form:

Find $u \in K$ and $u^{*} \in F(u)$ such that

$$
\left\langle u^{*}, v-u\right\rangle+\phi(v)-\phi(u)+J^{\circ}(u ; v-u) \geq 0, \quad \forall v \in K .
$$

The existence of solutions to this inequality was studied by Tang and Huang [34]. 
(c) If $T \equiv 0$ and $f \equiv 0$, then GVHVI is reduced to the following form:

Find $u \in K$ and $u^{*} \in F(u)$ such that

$$
\left\langle u^{*}, v-u\right\rangle+g(u, v)+J^{\circ}(u ; v-u) \geq 0, \quad \forall v \in K
$$

The existence of solutions to this inequality was studied by Wangkeeree and Preechasilp [36].

Inspired by previous works, we study the well-posedness for GVHVI, which generalizes many known works. Under relatively weak conditions, we establish some equivalence results and some metric characterizations for the strong and weak $\alpha$-well-posed GVHVI in the generalized sense. In particular, we present equivalence results on weak $\alpha$-wellposedness for GVHVI, which were considered by few authors.

This paper is organized as follows. In Sect. 2, we recall some basic preliminaries of singlevalued and set-valued mappings, metric concepts, Clarke's generalized directional derivative, and some classes of well-posedness for GVHVI. In Sect. 3, we show some equivalence results for the well-posedness for GVHVI and some corresponding metric characterizations. Theorems 3.3, 3.5, and 3.6 are the main results in this section. In the last section, we also present the well-posedness for a class of generalized mixed equilibrium problems.

\section{Preliminaries}

Let $R, R_{+}$, and $N$ be the sets of real numbers, nonnegative real numbers, and natural numbers, respectively. Let $X$ be a real Banach space with norm $\|\cdot\|_{X}$. Denote by $X^{*}$ its dual space and by $\langle\cdot, \cdot\rangle_{X}$ the duality pairing between $X^{*}$ and $X$. Let $X_{w}$ be the Banach space $X$ with weak topology.

Definition 2.1 Let $K$ be a nonempty subset of $X$. A function $f: K \rightarrow R$ is said to be

(i) convex on $K$ if for all finite subsets $\left\{u_{1}, \ldots, u_{n}\right\} \subset K$ and $\left\{\lambda_{1}, \ldots, \lambda_{n}\right\} \subset R_{+}$such that $\sum_{i=1}^{n} \lambda_{i}=1$ and $\sum_{i=1}^{n} \lambda_{i} u_{i} \in K$, we have

$$
f\left(\sum_{i=1}^{n} \lambda_{i} u_{i}\right) \leq \sum_{i=1}^{n} \lambda_{i} f\left(u_{i}\right)
$$

(ii) (weakly) upper semicontinuous (u.s.c. for short) at $u$ if for any sequence $\left\{u_{n}\right\}_{n \geq 1} \subset K$ with $\left(u_{n} \rightarrow u\right) u_{n} \rightarrow u$, we have

$$
\limsup _{n \rightarrow \infty} f\left(u_{n}\right) \leq f(u)
$$

(iii) (weakly) lower semicontinuous (l.s.c. for short) at $u$, if for any sequence $\left\{u_{n}\right\}_{n \geq 1} \subset K$ with $\left(u_{n} \rightarrow u\right) u_{n} \rightarrow u$, we have

$$
\liminf _{n \rightarrow \infty} f\left(u_{n}\right) \geq f(u) .
$$

The function $f$ is said to be (weakly) u.s.c. (l.s.c.) on $K$ if $f$ is (weakly) u.s.c. (l.s.c.) at all $u \in K$. 
Definition 2.2 ([20]) Let $K$ be a nonempty subset of $X$. An operator $\beta: K \rightarrow X$ is said to be affine if for any $u_{i} \in K(i=1,2, \ldots, n)$ and $\lambda_{i} \in[0,1]$ with $\sum_{i=1}^{n} \lambda_{i}=1$, we have

$$
\beta\left(\sum_{i=1}^{n} \lambda_{i} v_{i}\right)=\sum_{i=1}^{n} \lambda_{i} \beta\left(u_{i}\right)
$$

Definition 2.3 A set-valued operator $F: K \rightarrow P\left(X^{*}\right)$ is said to be

(i) lower semicontinuous (l.s.c.) at $u_{0}$ if for any $u_{0}^{*} \in F\left(u_{0}\right)$ and sequence $\left\{u_{n}\right\}_{n \geq 1} \subset K$ with $u_{n} \rightarrow u_{0}$, there exists a sequence $u_{n}^{*} \in F\left(u_{n}\right)$ that converges to $u_{0}^{*}$.

(ii) lower hemicontinuous (l.h.c.) if the restriction of $F$ to every line segment of $K$ is lower semicontinuous with respect to the weak topology in $X^{*}$.

Definition 2.4 A set-valued operator $F: K \rightarrow P\left(X^{*}\right)$ is said to be monotone if for all $u, v \in K$,

$$
\left\langle v^{*}-u^{*}, v-u\right\rangle \geq 0, \quad \forall u^{*} \in F(u), \forall v^{*} \in F(v)
$$

Definition 2.5 Let $S$ be a nonempty subset of $X$. The measure $\mu$ of noncompactness for the set $S$ is defined by

$$
\mu(S):=\inf \left\{\epsilon>0: S=\bigcup_{i=1}^{n} S_{i}, \operatorname{diam}\left|S_{i}\right|<\epsilon, i=1,2, \ldots, n\right\},
$$

where $\operatorname{diam}\left|S_{i}\right|$ is the diameter of the set $S_{i}$.

Now, let us recall the definitions of the Clarke generalized directional derivative and generalized gradient for a locally Lipschitz function $\varphi: X \rightarrow R$ (see $[6,10])$. The Clarke generalized directional derivative $\varphi^{0}(u ; v)$ of $\varphi$ at the point $u \in X$ in the direction $v \in X$ is defined as

$$
\varphi^{0}(u ; v):=\limsup _{\lambda \rightarrow 0^{+}, \zeta \rightarrow u} \frac{\varphi(\zeta+\lambda \nu)-\varphi(\zeta)}{\lambda} .
$$

The Clarke subdifferential or generalized gradient of $\varphi$ at $u \in X$, denoted by $\partial \varphi(u)$, is the subset of $X^{*}$ given by

$$
\partial \varphi(u):=\left\{u^{*} \in X^{*}: \varphi^{0}(u ; v) \geq\left\langle u^{*}, v\right\rangle_{X}, \forall v \in X\right\} .
$$

Lemma 2.6 ([6], Proposition 2.1.1) Let $\varphi: X \rightarrow R$ be locally Lipschitz of rank $L_{u}>0$ near $u$. Then

(i) $\varphi^{0}(u ; v)$ is u.s.c. as a function of $(u, v)$ and, as a function of $v$ alone, is Lipschitz of rank $L_{u}$ near $u$ on $X$ and satisfies

$$
\left|\varphi^{0}(u ; v)\right| \leq L_{u}\|v\|_{X}
$$

(ii) the gradient $\partial \varphi(u)$ is a nonempty, convex, and weakly* compact subset of $X^{*}$ bounded by a Lipschitz constant $L_{u}$ near $x$; 
(iii) for every $v \in X$, we have

$$
\varphi^{0}(u ; v)=\max \left\{\left\langle u^{*}, v\right\rangle \mid u^{*} \in \partial \varphi(u)\right\}
$$

We end this section with the notions of several classes of $\alpha$-approximating sequences and $\alpha$-well-posedness for GVHVI. Let $\alpha: X \rightarrow R_{+}$be a functional.

Definition 2.7 A sequence $\left\{u_{n}\right\}$ in $K$ is an $\alpha$-approximating sequence for GVHVI if there exist $\left\{u_{n}^{*}\right\}$ in $X^{*}$ with $u_{n}^{*} \in F\left(u_{n}\right)$ and a nonnegative sequence $\left\{\epsilon_{n}\right\}$ with $\epsilon_{n} \rightarrow 0$ as $n \rightarrow \infty$ such that, for every $n \in N$,

$$
\left\langle u_{n}^{*}+T u_{n}-f, v-u_{n}\right\rangle+g\left(u_{n}, v\right)+J^{\circ}\left(u_{n} ; v-u_{n}\right) \geq-\epsilon_{n} \alpha\left(v-u_{n}\right), \quad \forall v \in K .
$$

In particular, if $\alpha(\cdot)=\|\cdot\|_{X}$, then $\left\{u_{n}\right\}$ is said to be an approximating sequence for GVHVI.

Definition 2.8 GVHVI is said to be strongly (respectively, weakly) $\alpha$-well-posed if it has a unique solution $u$ and every $\alpha$-approximating sequence $\left\{u_{n}\right\}$ strongly (respectively, weakly) converges to $u$. In particular, if $\alpha(\cdot)=\|\cdot\|_{X}$, then GVHVI is said to be strongly (respectively, weakly) well-posed.

Definition 2.9 GVHVI is said to be strongly (respectively, weakly) $\alpha$-well-posed in the generalized sense if the solution set $\Gamma$ of GVHVI is nonempty and every $\alpha$-approximating sequence $\left\{u_{n}\right\}$ has a subsequence that strongly (respectively, weakly) converges to some point of $\Gamma$. In particular, if $\alpha(\cdot)=\|\cdot\|_{X}$, then GVHVI is said to be strongly (respectively, weakly) well-posed in the generalized sense.

Remark 2.10 Strong $\alpha$-well-posedness (in the generalized sense) implies weak $\alpha$-wellposedness (in the generalized sense), but the converse is not true in general.

\section{The characterizations of well-posedness for GVHVI}

In this section, we establish metric characterizations and derive some conditions under which GVHVI is strongly (weakly) $\alpha$-well-posed.

For any $\epsilon>0$, we define the following two sets:

$$
\begin{aligned}
\Omega_{\alpha}(\epsilon)= & \left\{u \in K: \exists u^{*} \in F(u) \text { such that }\left\langle u^{*}+T u-f, v-u\right\rangle+g(u, v)\right. \\
& \left.+J^{\circ}(u ; v-u) \geq-\epsilon \alpha(v-u), \forall v \in K\right\}
\end{aligned}
$$

and

$$
\begin{aligned}
\Phi_{\alpha}(\epsilon)= & \left\{u \in K:\left\langle v^{*}+T u-f, v-u\right\rangle+g(u, v)+J^{\circ}(u ; v-u)\right. \\
& \left.\geq-\epsilon \alpha(v-u), \forall v \in K, \forall v^{*} \in F(v)\right\} .
\end{aligned}
$$

Denote by $\Gamma$ the set of solutions to GVHVI. It is clear that $\Gamma=\Omega_{0}(\epsilon)$.

Lemma 3.1 Assume that:

(i) $K$ is a nonempty closed subset of a real Banach space $X$; 
(ii) $T: K \rightarrow X_{w}^{*}$ is continuous;

(iii) $g: K \times K \rightarrow R$ is u.s.c. with respect to the first variable;

(iv) $\alpha: X \rightarrow R_{+}$is such that $\liminf _{n \rightarrow \infty} \alpha\left(v_{n}\right) \leq \alpha(v)$ whenever $v_{n} \rightarrow v$.

Then, for every $\epsilon>0$, the set $\Phi_{\alpha}(\epsilon)$ is closed in $X$.

Proof Let $\left\{u_{n}\right\} \subset \Phi_{\alpha}(\epsilon)$ be s sequence such that $u_{n} \rightarrow u$ in $X$. Then $u \in K$, and, for all $v \in K$ and $v^{*} \in F(v)$,

$$
\left\langle v^{*}+T u_{n}-f, v-u_{n}\right\rangle+g\left(u_{n}, v\right)+J^{\circ}\left(u_{n} ; v-u_{n}\right) \geq-\epsilon \alpha\left(v-u_{n}\right) .
$$

By the assumptions and the properties of $J^{\circ}$ we have

$$
\begin{aligned}
& \left\langle v^{*}+T u-f, v-u\right\rangle+g(u, v)+J^{\circ}(u ; v-u) \\
& \quad \geq \limsup _{n \rightarrow \infty}\left[\left\langle v^{*}+T u_{n}-f, v-u_{n}\right\rangle+g\left(u_{n}, v\right)+J^{\circ}\left(u_{n} ; v-u_{n}\right)\right] \\
& \geq \limsup _{n \rightarrow \infty}-\epsilon \alpha\left(v-u_{n}\right) \\
& =-\epsilon \liminf _{n \rightarrow \infty} \alpha\left(v-u_{n}\right) \\
& \geq-\epsilon \alpha(v-u),
\end{aligned}
$$

and hence

$$
\left\langle v^{*}+T u-f, v-u\right\rangle+g(u, v)+J^{\circ}(u ; v-u)-\epsilon \alpha(v-u), \quad \forall v \in K, \forall v^{*} \in F(v),
$$

which shows that $u \in \Phi_{\alpha}(\epsilon)$.

\section{Lemma 3.2 Assume that:}

(i) $K$ is a nonempty convex subset of a real Banach space $X$;

(ii) $F: K \rightarrow P\left(X^{*}\right)$ is l.h.c. and monotone;

(iii) $g: K \times K \rightarrow R$ is convex with respect to the second variable;

(iv) $\alpha: X \rightarrow R_{+}$is convex with $\alpha(t v)=t \alpha(v)$ for all $t \geq 0$ and $v \in X$.

Then $\Omega_{\alpha}(\epsilon)=\Phi_{\alpha}(\epsilon)$ for all $\epsilon>0$.

Proof We first show that $\Omega_{\alpha}(\epsilon) \subset \Phi_{\alpha}(\epsilon)$. Indeed, take arbitrary $u \in \Omega_{\alpha}(\epsilon)$. Then there exists $u^{*} \in F(u)$ such that

$$
\left\langle u^{*}+T u-f, v-u\right\rangle+g(u, v)+J^{\circ}(u ; v-u) \geq-\epsilon \alpha(v-u), \quad \forall v \in K
$$

According to the monotonicity of $F$, we obtain

$$
\left\langle v^{*}+T u-f, v-u\right\rangle+g(u, v)+J^{\circ}(u ; v-u) \geq-\epsilon \alpha(v-u), \quad \forall v \in K, \forall v^{*} \in F(v)
$$

which means that $u \in \Phi_{\alpha}(\epsilon)$. Therefore $\Omega_{\alpha}(\epsilon) \subset \Phi_{\alpha}(\epsilon)$.

Now we show that $\Phi_{\alpha}(\epsilon) \subset \Omega_{\alpha}(\epsilon)$. Indeed, take arbitrary $u \in \Phi_{\alpha}(\epsilon)$. Then

$$
\left\langle v^{*}+T u-f, v-u\right\rangle+g(u, v)+J^{\circ}(u ; v-u) \geq-\epsilon \alpha(v-u), \quad \forall v \in K, \forall v^{*} \in F(v) .
$$


Since the set $K$ is convex, for any $v \in K$ and $\lambda \in[0,1]$, taking $v_{\lambda}:=\lambda v+(1-\lambda) u \in K$ in this inequality, we have

$$
\left\langle v_{\lambda}^{*}+T u-f, v_{\lambda}-u\right\rangle+g\left(u, v_{\lambda}\right)+J^{\circ}\left(u ; v_{\lambda}-u\right) \geq-\epsilon \alpha\left(v_{\lambda}-u\right), \quad \forall v_{\lambda}^{*} \in F\left(v_{\lambda}\right) .
$$

Then by (iii), (iv), and the properties of $J^{\circ}$ we obtain

$$
\left\langle v_{\lambda}^{*}+T u-f, v-u\right\rangle+g(u, v)+J^{\circ}(u ; v-u) \geq-\epsilon \alpha(v-u), \quad \forall v_{\lambda}^{*} \in F\left(v_{\lambda}\right) .
$$

Let $u^{*} \in F(u)$ be fixed, and let $v_{\lambda}^{*} \in F\left(v_{\lambda}\right)$ be such that $v_{\lambda}^{*} \rightarrow u^{*}$ in $X^{*}$ (the existence of such a sequence is ensured by the fact that $F$ is l.h.c.). Taking the limit as $\lambda \rightarrow 0$ in (3.1), we obtain

$$
\begin{aligned}
& \left\langle u^{*}+T u-f, v-u\right\rangle+g(u, v)+J^{\circ}(u ; v-u) \\
& \quad=\lim _{\lambda \rightarrow 0}\left[\left\langle v_{\lambda}^{*}+T u-f, v-u\right\rangle+g(u, v)+J^{\circ}(u ; v-u)\right] \\
& \geq-\epsilon \alpha(v-u),
\end{aligned}
$$

which implies that $u \in \Omega_{\alpha}(\epsilon)$. The proof is complete.

The following result is a consequence of Lemmas 3.1 and 3.2.

\section{Theorem 3.3 Assume that:}

(i) $K$ is a nonempty closed convex subset of a real Banach space $X$;

(ii) $F: K \rightarrow P\left(X^{*}\right)$ is l.h.c. and monotone;

(iii) $T: K \rightarrow X_{w}^{*}$ is continuous;

(iv) $g: K \times K \rightarrow R$ is u.s.c. with respect to the first variable and convex with respect to the second variable;

(v) $\alpha: X \rightarrow R_{+}$is continuous and convex with $\alpha(t v)=t \alpha(v)$ for all $t \geq 0$ and $v \in X$.

Then $\Omega_{\alpha}(\epsilon)=\Phi_{\alpha}(\epsilon)$ is closed in X for all $\epsilon>0$. Moreover, $\Gamma=\Omega_{0}(\epsilon)=\Phi_{0}(\epsilon)$, that is, GVHVI is equivalent to the following problem:

Find $u \in K$ such that

$$
\left\langle v^{*}+T u-f, v-u\right\rangle+g(u, v)+J^{\circ}(u ; v-u) \geq 0, \quad \forall v \in K, v^{*} \in F(v) .
$$

Theorem 3.4 GVHVI is strongly $\alpha$-well-posed if and only if $\Gamma$ is nonempty and

$$
\lim _{\epsilon \rightarrow 0} \operatorname{diam}\left(\Omega_{\alpha}(\epsilon)\right)=0
$$

Proof The proof is similar to that of Theorem 4.3 in [26] by the assumptions of $g$.

Theorem 3.5 Assume that all the assumptions of Theorem 3.3 are satisfied. Then GVHVI is strongly $\alpha$-well-posed if and only if

$$
\Omega_{\alpha}(\epsilon) \neq \emptyset \quad \forall \epsilon \geq 0 \quad \text { and } \quad \lim _{\epsilon \rightarrow 0} \operatorname{diam}\left(\Omega_{\alpha}(\epsilon)\right)=0
$$


Proof Suppose that GVHVI is strongly $\alpha$-well-posed. Then GVHVI has a unique solution $u \in K$, and thus $\Gamma \neq \emptyset$. Now, we prove that (3.2) holds. Clearly, $\Omega_{\alpha}(\epsilon) \supset \Gamma \neq \emptyset$. For the second part of (3.2), arguing by contradiction, let us assume that $\operatorname{diam}\left(\Omega_{\alpha}(\epsilon)\right)$ does not tend to 0 as $\epsilon \rightarrow 0$. Thus for any nonnegative sequence $\left\{\epsilon_{n}\right\}$ with $\epsilon_{n} \rightarrow 0$ as $n \rightarrow \infty$, there exists a constant $\beta>0$ such that, for each $n \in N$, there exist $u_{n}^{(1)}, u_{n}^{(2)} \in \Omega_{\alpha}\left(\epsilon_{n}\right)$ satisfying

$$
\left\|u_{n}^{(1)}-u_{n}^{(2)}\right\|>\beta>0
$$

Since $u_{n}^{(1)}, u_{n}^{(2)} \in \Omega_{\alpha}\left(\epsilon_{n}\right)$, we know that the sequences $\left\{u_{n}^{(1)}\right\}$ and $\left\{u_{n}^{(2)}\right\}$ are both $\alpha$ approximating sequences of GVHVI, and thus

$$
\lim _{n \rightarrow} u_{n}^{(1)}=\lim _{n \rightarrow} u_{n}^{(2)}=u
$$

From (3.3) and (3.4) we have

$$
0<\beta<\left\|u_{n}^{(1)}-u_{n}^{(2)}\right\| \leq\left\|u_{n}^{(1)}-u\right\|+\left\|u_{n}^{(2)}-u\right\| \rightarrow 0
$$

which is a contradiction.

Conversely, assume that condition (3.2) holds. Let $\left\{u_{n}\right\}$ in $K$ be an $\alpha$-approximating sequence for GVHVI. Then, there exist $\left\{u_{n}^{*}\right\}$ in $X^{*}$ with $u_{n}^{*} \in F\left(u_{n}\right)$ and a nonnegative sequence $\left\{\epsilon_{n}\right\}$ with $\epsilon_{n} \rightarrow 0$ as $n \rightarrow \infty$ such that, for every $n \in N$,

$$
\left\langle u_{n}^{*}+T u_{n}-f, v-u_{n}\right\rangle+g\left(u_{n}, v\right)+J^{\circ}\left(u_{n} ; v-u_{n}\right) \geq-\epsilon_{n} \alpha\left(v-u_{n}\right), \quad \forall v \in K,
$$

that is, $u_{n} \in \Omega_{\alpha}\left(\epsilon_{n}\right)$ for all $n \in N$. By condition (3.2) we deduce that the sequence $\left\{u_{n}\right\}$ is a Cauchy sequence, and so $\left\{u_{n}\right\}$ converges strongly to some point $u \in K$. Let us show that $u \in K$ is a solution for GVHVI. By the monotonicity of $F$ we obtain that, for every $n \in N$,

$$
\begin{aligned}
& \left\langle v^{*}+T u_{n}-f, v-u_{n}\right\rangle+g\left(u_{n}, v\right)+J^{\circ}\left(u_{n} ; v-u_{n}\right) \\
& \quad \geq\left\langle u_{n}^{*}+T u_{n}-f, v-u_{n}\right\rangle+g\left(u_{n}, v\right)+J^{\circ}\left(u_{n} ; v-u_{n}\right) \\
& \geq-\epsilon_{n} \alpha\left(v-u_{n}\right), \quad \forall v \in K, v^{*} \in F(v) .
\end{aligned}
$$

By the assumptions we obtain that

$$
\begin{aligned}
& \left\langle v^{*}+T u-f, v-u\right\rangle+g(u, v)+J^{\circ}(u ; v-u) \\
& \quad \geq \limsup _{n \rightarrow \infty}\left[\left\langle v^{*}+T u_{n}-f, v-u_{n}\right\rangle+g\left(u_{n}, v\right)+J^{\circ}\left(u_{n} ; v-u_{n}\right)\right] \\
& \geq \limsup _{n \rightarrow \infty}-\epsilon_{n} \alpha\left(v-u_{n}\right) \\
& =\limsup _{n \rightarrow \infty} \alpha\left(-\epsilon_{n}\left(v-u_{n}\right)\right) \\
& =0
\end{aligned}
$$

which implies that

$$
\left\langle v^{*}+T u-f, v-u\right\rangle+g(u, v)+J^{\circ}(u ; v-u) \geq 0, \quad \forall v \in K, \forall v^{*} \in F(v) .
$$


It follows from Theorem 3.3 that there exists $u^{*} \in F(u)$ such that

$$
\left\langle u^{*}+T u-f, v-u\right\rangle+g(u, v)+J^{\circ}(u ; v-u) \geq 0, \quad \forall v \in K
$$

Then $u \in K$ is a solution of GVHVI.

Finally, we prove that the solution $u$ is unique. If there exists another solution $u^{\prime} \in K$, then $u, u_{1} \in \Omega_{\alpha}(\epsilon)$ for all $\epsilon>0$, and

$$
0<\left\|u-u^{\prime}\right\| \leq \operatorname{diam}\left(\Omega_{\alpha}(\epsilon)\right) \rightarrow 0 \quad \text { as } \epsilon \rightarrow 0
$$

which is a contradiction. This completes the proof.

\section{Theorem 3.6 Assume that:}

(i) $K$ is a nonempty closed convex subset of a real reflexive Banach space $X$;

(ii) $F: K \rightarrow P\left(X^{*}\right)$ is l.h.c. and monotone;

(iii) $T: K \rightarrow X^{*}$ is compact;

(iv) $g: K \times K \rightarrow R$ is weakly u.s.c. with respect to the first variable and convex with respect to the second variable;

(v) $\lim \sup _{n \rightarrow \infty} J^{\circ}\left(u_{n} ; v-u_{n}\right) \leq J^{\circ}(u ; v-u)$ for all $v \in X$ whenever $u_{n} \rightarrow u$ as $n \rightarrow \infty$;

(vi) $\alpha: X \rightarrow R_{+}$is a continuous and convex functional with $\alpha(t v)=t \alpha(v)$ for all $t \geq 0$ and $v \in X$.

Then GVHVI is weakly $\alpha$-well-posed if and only if GVHVI has a unique solution and there exists $\epsilon_{0}>0$ such that $\Omega_{\alpha}\left(\epsilon_{0}\right)$ is nonempty and bounded.

Proof The necessity is obvious. We now prove the sufficiency. Let $\left\{u_{n}\right\}$ be an $\alpha$ approximating sequence for GVHVI. Then, there exist $\left\{u_{n}^{*}\right\}$ in $X^{*}$ with $u_{n}^{*} \in F\left(u_{n}\right)$ and a nonnegative sequence $\left\{\epsilon_{n}\right\}$ with $\epsilon_{n} \rightarrow 0$ as $n \rightarrow \infty$ such that, for every $n \in N$,

$$
\left\langle u_{n}^{*}+T u_{n}-f, v-u_{n}\right\rangle+g\left(u_{n}, v\right)+J^{\circ}\left(u_{n} ; v-u_{n}\right) \geq-\epsilon_{n} \alpha\left(v-u_{n}\right)
$$

for all $v \in K$. We claim that the sequence $\left\{u_{n}\right\}$ is bounded in $X$. Indeed, since $\Omega_{\alpha}\left(\epsilon_{0}\right)$ is bounded and $\Omega_{\alpha}(\epsilon) \subset \Omega_{\alpha}\left(\epsilon_{0}\right)$ for all $\epsilon \in\left(0, \varepsilon_{0}\right)$, there exists $n_{0} \in N$ such that $\epsilon_{n_{0}} \in\left(0, \varepsilon_{0}\right)$ and $u_{n} \in \Omega_{\alpha}\left(\epsilon_{0}\right)$ for all $n \geq n_{0}$, which shows that $\left\{u_{n}\right\}$ is bounded in $X$.

Since the Banach space $X$ is reflexive, we can choose a subsequence of $\left\{u_{n}\right\}$, denoted by $\left\{u_{n}\right\}$ again, such that $u_{n} \rightarrow \bar{u}$ as $n \rightarrow \infty$ for some $\bar{u} \in X$. Let us show that $\bar{u} \in K$ is a solution for GVHVI. Obviously, $\bar{u} \in K$. By the monotonicity of $F$ we obtain that

$$
\begin{aligned}
& \left\langle v^{*}+T u_{n}-f, v-u_{n}\right\rangle+g\left(u_{n}, v\right)+J^{\circ}\left(u_{n} ; v-u_{n}\right) \\
& \geq\left\langle u_{n}^{*}+T u_{n}-f, v-u_{n}\right\rangle+g\left(u_{n}, v\right)+J^{\circ}\left(u_{n} ; v-u_{n}\right) \\
& \geq-\epsilon_{n} \alpha\left(v-u_{n}\right), \quad \forall v \in K, v^{*} \in F(v), \forall n \in N .
\end{aligned}
$$

By the assumptions, we obtain that

$$
\begin{aligned}
& \left\langle v^{*}+T \bar{u}-f, v-\bar{u}\right\rangle+g(\bar{u}, v)+J^{\circ}(\bar{u} ; v-\bar{u}) \\
& \quad \geq \limsup _{n \rightarrow \infty}\left[\left\langle v^{*}+T u_{n}-f, v-u_{n}\right\rangle+g\left(u_{n}, v\right)+J^{\circ}\left(u_{n} ; v-u_{n}\right)\right]
\end{aligned}
$$




$$
\begin{aligned}
& \geq \limsup _{n \rightarrow \infty}-\epsilon_{n} \alpha\left(v-u_{n}\right) \\
& =\limsup _{n \rightarrow \infty} \alpha\left(-\epsilon_{n}\left(v-u_{n}\right)\right) \\
& =0,
\end{aligned}
$$

which implies that

$$
\left\langle v^{*}+T \bar{u}-f, v-\bar{u}\right\rangle+g(\bar{u}, v)+J^{\circ}(\bar{u} ; v-\bar{u}) \geq 0, \quad \forall v \in K, \forall v^{*} \in F(v) .
$$

It follows from Theorem 3.3 that there exists $\bar{u}^{*} \in F(\bar{u})$ such that

$$
\left\langle u^{*}+T \bar{u}-f, v-\bar{u}\right\rangle+g(\bar{u}, v)+J^{\circ}(\bar{u} ; v-\bar{u}) \geq 0, \quad \forall v \in K,
$$

Therefore $\bar{u} \in K$ is a solution to problem GVHVI, and so we get that GVHVI is weakly $\alpha$-well-posed by the uniqueness of the solution to problem GVHVI. This completes the proof.

Remark 3.7 In the theorem, condition (v) can be found in [30], and the condition that there exists $\epsilon_{0}>0$ such that $\Omega_{\alpha}\left(\epsilon_{0}\right)$ is nonempty and bounded can be replaced by the conditions that $K$ is bounded or that there exists $n_{0} \in N$ such that, for every $u \in K \backslash B_{n_{0}}$, there exists $v \in K$ with $\|v\|<\|u\|$ such that

$$
\sup _{u^{*} \in F(u)}\left\langle u^{*}+T u-f, v-u\right\rangle+g(u, v)+J^{\circ}(u ; v-u) \leq-\frac{1}{n_{0}} .
$$

See $[34,36,39]$ for more detail.

Next, we give some equivalence results for the strong $\alpha$-posedness in the generalized sense.

Theorem 3.8 Assume that all the assumptions of Theorem 3.5 are satisfied. Then GVHVI is strongly $\alpha$-well-posed in the generalized sense if and only if $\Gamma$ is nonempty compact and

$$
\lim _{\epsilon \rightarrow 0} e\left(\Omega_{\alpha}(\epsilon), \Gamma\right)=0
$$

where $e(A, B):=\sup _{a \in A} d(a, B)$ with $d(a, B):=\inf _{b \in B}\|a-b\|$.

Proof The proof is similar to that of Theorem 5.1 in [26] by the assumptions of $g$.

Theorem 3.9 Assume that all the assumptions of Theorem 3.5 are satisfied. Then GVHVI is strongly $\alpha$-well-posed in the generalized sense if and only if

$$
\Omega_{\alpha}(\epsilon) \neq \emptyset, \quad \forall \epsilon>0, \quad \text { and } \quad \lim _{\epsilon \rightarrow 0} \mu\left(\Omega_{\alpha}(\epsilon)\right)=0
$$

Proof The proof is similar to that of Theorem 3.2 in [3] by the assumptions of $g$. 
Theorem 3.10 Assume that all the assumptions of Theorem 3.6 are satisfied. Then GVHVI is weakly $\alpha$-well-posed in the generalized sense if and only if there exists $\epsilon_{0}>0$ such that $\Omega_{\alpha}\left(\epsilon_{0}\right)$ is nonempty and bounded.

Proof The proof is similar to that of Theorem 3.6 by the assumptions of $g$.

\section{Well-posedness for GMEP}

In this section, we consider the following generalized mixed equilibrium problem (GMEP):

Find $u \in K$ such that, for some $u^{*} \in F(u)$,

$$
\left\langle u^{*}, \eta(u, v)\right\rangle+\langle T u-f, v-u\rangle+g(u, v)+h(u, v) \geq 0, \quad \forall v \in K,
$$

where $\eta: K \times K \rightarrow X$ is an operator. The existence of solutions to this problem when $T \equiv 0$ and $f \equiv 0$ can be found in [25].

To study GMEP, we introduce the concept of $\eta$-monotonicity (see $[7,8]$ ).

Definition 4.1 Let $F: K \rightarrow P\left(X^{*}\right)$ be a set-valued operator. $F$ is said to be $\eta$-monotone if there exists a function $\eta: K \times K \rightarrow X$ such that, for all $u, v \in K$,

$$
\left\langle v^{*}-u^{*}, \eta(u, v)\right| \geq 0, \quad \forall u^{*} \in F(u), \forall v^{*} \in F(v) .
$$

Remark 4.2 If $\eta(u, v)=v-u$ for all $u, v \in X$, then (4.1) becomes

$$
\left\langle v^{*}-u^{*}, v-u\right\rangle \geq 0, \quad \forall u^{*} \in F(u), \forall v^{*} \in F(v)
$$

that is, $F$ is monotone.

For any $\epsilon>0$, we define the following two sets:

$$
\begin{aligned}
\Omega_{\eta, \alpha}(\epsilon)= & \left\{u \in K: \exists u^{*} \in F(u) \text { such that }\left\langle u^{*}, \eta(u, v)\right\rangle+\langle T u-f, v-u\rangle+g(u, v)\right. \\
& +h(u, v)) \geq-\epsilon \alpha(v-u), \forall v \in K\}
\end{aligned}
$$

and

$$
\begin{aligned}
\Phi_{\eta, \alpha}(\epsilon)= & \left\{u \in K:\left\langle v^{*}, \eta(u, v)\right\rangle+\langle T u-f, v-u\rangle+g(u, v)\right. \\
& \left.+h(u, v) \geq-\epsilon \alpha(v-u), \forall v \in K, \forall v^{*} \in F(v)\right\} .
\end{aligned}
$$

Denote by $\Gamma_{\eta}$ the set of solutions to GMEP. It is clear that $\Gamma=\Omega_{0}(\epsilon)$.

We can obtain similar results.

Theorem 4.3 Assume that all the assumptions of Theorem 3.3 are satisfied and, in addition, $\eta: K \times K \rightarrow X$ is continuous on $K \times K$ with $\eta(u, u)=0$ for any $u \in K$ and affine with respect to the first variable. Let $h: K \times K \rightarrow R$ be such that:

(i) $h(u, u)=0$ for all $u \in X$,

(ii) for all $v \in K, h(\cdot, v)$ is u.s.c.,

(iii) for all $u \in K, h(u, \cdot)$ is convex. 
Then $\Omega_{\eta, \alpha}(\epsilon)=\Phi_{\eta, \alpha}(\epsilon)$ is closed in X for all $\epsilon>0$. Moreover, $\Gamma_{\eta}=\Omega_{\eta, 0}(\epsilon)=\Phi_{\eta, 0}(\epsilon)$, that is, GMEP is equivalent to the following problem:

Find $u \in K$ such that

$$
\left\langle v^{*}+T u-f, \eta(u, v)\right\rangle+g(u, v)+h(u, v) \geq 0, \quad \forall v \in K, v^{*} \in F(v) .
$$

Theorem 4.4 Assume that all the assumptions of Theorem 3.5 are satisfied and, in addition, $\eta: K \times K \rightarrow X$ is continuous on $K \times K$ with $\eta(u, u)=0$ for any $u \in K$ and affine with respect to the first variable. Let $h: K \times K \rightarrow R$ be such that:

(i) $h(u, u)=0$ for all $u \in X$,

(ii) for all $v \in K, h(\cdot, v)$ is u.s.c.,

(iii) for all $u \in K, h(u, \cdot)$ is convex.

Then GMVHVI is strongly $\alpha$-well-posed if and only if

$$
\Omega_{\eta, \alpha}(\epsilon) \neq \emptyset, \quad \forall \epsilon \geq 0, \quad \text { and } \quad \lim _{\epsilon \rightarrow 0} \operatorname{diam}\left(\Omega_{\eta, \alpha}(\epsilon)\right)=0
$$

Theorem 4.5 Assume that all the assumptions of Theorem 3.6 are satisfied and, in addition, $\eta: K \times K \rightarrow X$ is continuous on $K \times K$ with $\eta(u, u)=0$ for any $u \in K$ and affine with respect to the first variable. Let $h: K \times K \rightarrow R$ be such that:

(i) $h(u, u)=0$ for all $u \in X$,

(ii) for all $v \in K, h(\cdot, v)$ is weakly u.s.c.,

(iii) for all $u \in K, h(u, \cdot)$ is convex.

Then GMEP is weakly $\alpha$-well-posed if and only if GMEP has a unique solution and there exists $\epsilon_{0}>0$ such that $\Omega_{\alpha}\left(\epsilon_{0}\right)$ is nonempty and bounded.

Theorem 4.6 Assume that all the assumptions of Theorem 3.5 are satisfied and, in addition, $\eta: K \times K \rightarrow X$ is continuous on $K \times K$ with $\eta(u, u)=0$ for any $u \in K$ and affine with respect to the first variable. Let $h: K \times K \rightarrow R$ is such that:

(i) $h(u, u)=0$ for all $u \in X$,

(ii) for all $v \in K, h(\cdot, v)$ is u.s.c.,

(iii) for all $u \in K, h(u, \cdot)$ is convex.

Then GMEP is strongly $\alpha$-well-posed in the generalized sense if and only if

$$
\Omega_{\eta, \alpha}(\epsilon) \neq \emptyset, \quad \forall \epsilon>0, \quad \text { and } \quad \lim _{\epsilon \rightarrow 0} \mu\left(\Omega_{\eta, \alpha}(\epsilon)\right)=0 .
$$

Theorem 4.7 Assume that all the assumptions of Theorem 3.6 are satisfied and, in addition, $\eta: K \times K \rightarrow X$ is continuous on $K \times K$ with $\eta(u, u)=0$ for any $u \in K$ and affine with respect to the first variable. Let $h: K \times K \rightarrow R$ be such that:

(i) $h(u, u)=0$ for all $u \in X$,

(ii) for all $v \in K, h(\cdot, v)$ is weakly u.s.c.,

(iii) for all $u \in K, h(u, \cdot)$ is convex.

Then GMEP is weakly $\alpha$-well-posed in the generalized sense if and only if there exists $\epsilon_{0}>0$ such that $\Omega_{\alpha}\left(\epsilon_{0}\right)$ is nonempty and bounded.

\section{Conclusion}

In this paper, inspired by the previous works, we study the well-posedness for GVHVI. Under relatively weak conditions for the data $F, T, g$, $J$ (see Theorems 3.3 and 3.6), we 
provide some equivalence results for the strong and weak $\alpha$-well-posed GVHVI in the generalized sense. Our results generalize and improve many known results and can be applied to many other problems.

Funding

The work was supported by the National Natural Science Foundation of China Grant No. 11361009 and the High level innovation teams and distinguished scholars in Guangxi Universities.

\section{Competing interests}

The author declares to have no competing interests.

Authors' contributions

The author read and approved the final manuscript.

\section{Publisher's Note}

Springer Nature remains neutral with regard to jurisdictional claims in published maps and institutional affiliations.

Received: 14 April 2018 Accepted: 13 July 2018 Published online: 24 July 2018

\section{References}

1. Blum, E., Oettli, W.: From optimization and variational inequalities to equilibrium problems. Math. Stud. 63, 123-145 (1994)

2. Ceng, L.-C., Hadjisavvas, N., Schaible, S., Yao, J.C.: Well-posedness for mixed quasivariational-like inequalities. J. Optim. Theory Appl. 139, 109-125 (2008)

3. Ceng, L.-C., Lur, Y.Y., Wen, C.F.: Well-posedness for generalized variational-hemivariational inequalities with perturbations in reflexive Banach spaces. Tamkang J. Math. 48(4), 345-364 (2017)

4. Ceng, L.-C., Yao, J.C.: Well-posedness of generalized mixed variational inequalities inclusion problems and fixed point problem. Nonlinear Anal. TMA 69, 4585-4603 (2008)

5. Cho, S.Y.: Strong convergence analysis of a hybrid algorithm for nonlinear operators in a Banach space. J. Appl. Anal. Comput. 8, 19-31 (2018)

6. Clarke, F.H.: Optimization and Nonsmooth Analysis. Wiley, New York (1983)

7. Costea, N.: Existence and uniqueness results for a class of quasi-hemivariational inequalities. J. Math. Anal. Appl. 373, 305-311 (2011)

8. Costea, N., Lupu, C.: On a class of variational-hemivariational inequalities involving set valued mappings. Adv. Pure Appl. Math. 1, 233-246 (2010)

9. Darabi, M., Zafarani, J.: Hadamard well-posedness for vector parametric equilibrium problems. J. Nonlinear Var. Anal. 1, 281-295 (2017)

10. Denkowski, Z., Migórski, S., Papageorgiou, N.S.: An Introduction to Nonlinear Analysis: Theory. Kluwer Academic, Boston (2003)

11. Fan, K.: Some properties of convex sets related to fixed point theorems. Math. Ann. 266, 519-537 (1984)

12. Fang, Y.P., Huang, N.J.: Variational-like inequalities with generalized monotone mappings in Banach spaces. J. Optim. Theory Appl. 118, 327-338 (2003)

13. Fang, Y.P., Huang, N.J., Yao, J.C.: Well-posedness of mixed variational inequalities, inclusion problems and fixed-point problems. J. Glob. Optim. 41, 117-133 (2008)

14. Fang, Y.P., Huang, N.J., Yao, J.C.: Well-posedness by perturbations of mixed variational inequalities in Banach spaces. Eur. J. Oper. Res. 201, 682-692 (2010)

15. Goeleven, D., Mentagui, D.: Well-posed hemivariational inequalities. Numer. Funct. Anal. Optim. 16, $909-921$ (1995)

16. Guu, S.M., Li, J.: Vector variational-like inequalities with generalized bifunctions defined on nonconvex sets. Nonlinear Anal. 71, 2847-2855 (2009)

17. Huang, X.X., Yang, X.Q.: Generalized Levitin-Polyak well-posedness in constrained optimization. SIAM J. Optim. 17, 243-258 (2006)

18. Khakrah, E., Razani, A., Mirzaei, R., Oveisiha, M.: Some metric characterizations of well-posedness for hemivariational-like inequalities. J. Nonlinear Funct. Anal. 2017, Article ID 44 (2017)

19. Kimura, K., Liou, Y.C., Wu, S.Y., Yao, J.C.: Well-posedness for parametric vector equilibrium problems with applications. J. Ind. Manag. Optim. 4, 313-327 (2008)

20. Kristály, A., Rădulescu, V., Varga, C.: Variational Principles in Mathematical Physics, Geometry, and Economics: Qualitative Analysis of Nonlinear Equations and Unilateral Problems. Encylopedia of Mathematics, vol. 136. Cambridge University Press, Cambridge (2010)

21. Lemaire, B.: Well-posedness conditioning and regularization of minimization inclusion and fixed point problems. Pliska Stud. Math. Bulgar. 12, 71-84 (1998)

22. Li, X.B., Xia, F.Q.: Levitin-Polyak well-posedness of a generalized mixed variational inequality in Banach spaces. Nonlinear Anal. TMA 75, 2139-2153 (2012)

23. Lin, L.J., Chuang, C.S.: Well-posedness in the generalized sense for variational inclusion and disclusion problems and well-posedness for optimization problems with constraint. Nonlinear Anal. 70, 3609-3617 (2009)

24. Liu, Z.H., Migórski, S., Zeng, B.: Existence results and optimal control for a class of quasi mixed equilibrium problems involving the (f,g,h)-quasimonotonicity. Appl. Math. Optim. (2017). https://doi.org/10.1007/s00245-017-9431-3

25. Liu, Z.H., Zeng, B.: Existence results for a class of hemivariational inequalities involving the stable $(g, f, \alpha)$-quasimonotonicity. Topol. Methods Nonlinear Anal. 47(1), 195-217 (2016) 
26. Liu, Z.H., Zeng, S.D., Zeng, B.: Well-posedness for mixed quasi-variational hemivariational inequalities. Topol. Methods Nonlinear Anal. 47(2), 561-578 (2016)

27. Long, X.J., Huang, N.J.: Metric characterizations of $\alpha$-well-posedness for symimum problems with applications to variational inequalities. Numer. Funct. Anal. Optim. 3, 461-476 (1981)

28. Lucchetti, R., Patrone, F.: A characterization of Tykhonov well-posedness for minimum problems with applications to variational inequalities. Numer. Funct. Anal. Optim. 3, 461-476 (1981)

29. Migórski, S., Ochal, A., Sofonea, M.: Nonlinear Inclusions and Hemivariational Inequalities, Models and Analysis of Contact Problems. Advances in Mechanics and Mathematics, vol. 26. Springer, New York (2013)

30. Migórski, S., Ochal, A., Sofonea, M.: A class of variational-hemivariational inequalities in reflexive Banach spaces. J. Elast. 127, 151-178 (2017)

31. Naniewicz, Z., Panagiotopoulos, P.D.: Mathematical Theory of Hemivariational Inequalities and Applications. Dekker, New York (1995)

32. Panagiotopoulos, P.D.: Nonconvex energy functions, hemivariational inequalities and substationarity principles. Acta Mech. 48, 111-130 (1983)

33. Qin, X., Yao, J.C.: Projection splitting algorithms for nonself operators. J. Nonlinear Convex Anal. 18, 925-935 (2017)

34. Tang, G.J., Huang, N.J.: Existence theorems of the variational-hemivariational inequalities. J. Glob. Optim. 56, 605-622 (2013)

35. Tikhonov, A.N.: On the stability of the functional optimization problem. USSR Comput. Math. Math. Phys. 6, 28-33 (1966)

36. Wangkeeree, R., Preechasilp, P.: Existence theorems of the hemivariational inequality governed by a multi-valued map perturbed with a nonlinear term in Banach spaces. J. Glob. Optim. 57, 1447-1464 (2013)

37. Xiao, Y.B., Huang, N.J.: Well-posedness for a class of variational-hemivariational inequalities with perturbations. J. Optim. Theory Appl. 151, 33-51 (2011)

38. Xiao, Y.B., Huang, N.J., Wong, M.M.: Well-posedness of hemivariational inequalities and inclusion problems. Taiwan. J. Math. 15, 1261-1276 (2011)

39. Zhang, Y.L., He, Y.R.: The hemivariational inequalities for an upper semicontinuous set-valued mapping. J. Optim. Theory Appl. 156, 716-725 (2013)

\section{Submit your manuscript to a SpringerOpen ${ }^{\circ}$ journal and benefit from:}

- Convenient online submission

Rigorous peer review

- Open access: articles freely available online

- High visibility within the field

- Retaining the copyright to your article

Submit your next manuscript at $\gg$ springeropen.com 